\title{
A NEW PROOF OF A LEMMA BY PHELPS
}

\author{
ANTONIA E. CARDWELL
}

Received 7 December 2005; Revised 3 May 2006; Accepted 7 May 2006

We give a different proof of a lemma by Phelps (1960) which asserts, roughly speaking, that if two norm-one functionals $f$ and $g$ have their hyperplanes $f^{-1}(0)$ and $g^{-1}(0)$ sufficiently close together, then either $\|f-g\|$ or $\|f+g\|$ must be small. We also extend this result to a complex Banach space.

Copyright (c) 2006 Hindawi Publishing Corporation. All rights reserved.

In 1960 in [2], Phelps proved the following lemma.

Lemma 1. Suppose that $E$ is a real normed linear space and that $\epsilon>0$. If $f, g \in S^{*}$ are such that $f^{-1}(0) \cap \mathcal{U} \subset g^{-1}[-\epsilon / 2, \epsilon / 2]$, then either $\|f-g\| \leq \epsilon$ or $\|f+g\| \leq \epsilon$. (Here, $\mathcal{U}$ represents the unit ball of $E$ and $S^{*}$ is the unit sphere of $E^{*}$.)

This lemma was then used the following year as a crucial step in the proof of the well-known Bishop-Phelps theorem [1] that every Banach space is subreflexive; in other words, every functional on a Banach space $E$ can be approximated by a norm-attaining functional on the same space. The original proof of this lemma uses the Hahn-Banach theorem and is therefore fairly abstract.

In this note, we present an alternate proof for Lemma 1 stated above. This proof gives a geometric argument while extending the lemma to a complex Banach space. Lemma 1 is shown to be a special case when the bound of $\epsilon$ on $\|f \pm g\|$ is replaced by $5 \epsilon$. This replacement does not affect the fundamental conclusion of Lemma 1.

We now state the extended lemma.

Lemma 2. Let $X$ be a complex Banach space and let $\epsilon$ be such that $0<\epsilon<1 / 2$. Let $\varphi, \psi \in$ $X^{*},\|\varphi\|=\|\psi\|=1$. Suppose that for all $x \in X$ with $\|x\| \leq 1$ and $\varphi(x)=0$, it holds that $|\psi(x)| \leq \epsilon$. Then there is some complex number $\alpha$ such that $|\alpha|=1$ and $\|\varphi-\alpha \psi\| \leq 5 \epsilon$.

It will be shown that if $\varphi$ and $\psi$ are real-valued functionals on a real Banach space $X$, then $\alpha$ will in fact be either 1 or -1 , thus proving the amended original result.

We now prove Lemma 2. 
Proof. Let $e \in X$ be such that $\|e\|=1$ and $|\varphi(e)| \geq 1-\epsilon / 4$. We will first show that $|\psi(e)|$ $\geq 1-(5 / 2) \epsilon$. To see this, let $f \in X$ such that $\|f\|=1$ and $|\psi(f)| \geq 1-\epsilon / 4$. Let $k=$ $1-\epsilon / 4$ and let $t=\varphi(f) / \varphi(e)$. Then $0 \leq|t| \leq 1 /(1-\epsilon / 4)=1 / k \leq 8 / 7$ and if we take $w=(k /(k+1))(f-t e)$, then $\|w\| \leq(k /(k+1))(\|f\|+|t|\|e\|) \leq(k /(k+1))(1+1 / k)=1$. Moreover,

$$
\varphi(w)=\frac{k}{k+1}\left(\varphi(f)-\frac{\varphi(f)}{\varphi(e)} \varphi(e)\right)=0
$$

so we have

$$
\begin{aligned}
\epsilon & \geq|\psi(w)|=\frac{k}{k+1}|\psi(f)-t \psi(e)| \\
& \geq \frac{k}{k+1}|| \psi(f)|-| t|| \psi(e)|| \\
& \geq \frac{k}{k+1}(|\psi(f)|-|t||\psi(e)|) .
\end{aligned}
$$

Thus

$$
\frac{1}{k}|\psi(e)| \geq|t||\psi(e)| \geq|\psi(f)|-\frac{k+1}{k} \epsilon \geq\left(1-\frac{\epsilon}{4}\right)-\frac{k+1}{k} \epsilon=k-\frac{k+1}{k} \epsilon .
$$

This gives

$$
|\psi(e)| \geq k^{2}-(k+1) \epsilon=\left(1-\frac{\epsilon}{4}\right)^{2}-\left(2-\frac{\epsilon}{4}\right) \epsilon=1-\frac{\epsilon}{2}+\frac{\epsilon^{2}}{16}-2 \epsilon+\frac{\epsilon^{2}}{4} \geq 1-\frac{5}{2} \epsilon
$$

as required. Notice that, if $\varphi$ and $\psi$ are real valued, the above still holds.

Now, there exist $\beta, \gamma \in \mathbb{C}$ such that $|\beta|=|\gamma|=1, \beta \varphi(e) \in[1-\epsilon / 4,1] \subset \mathbb{R}$, and $\gamma \psi(e) \in[1-5 \epsilon / 2,1] \subset \mathbb{R}$; and so $|\beta \varphi(e)-\gamma \psi(e)| \leq 5 \epsilon / 2$.

Let $x \in X$ be such that $\|x\| \leq 1$ and write $x=\lambda e+y$, where $\lambda=\varphi(x) / \varphi(e)$ and $y=$ $x-\lambda e$. Then $|\lambda| \leq|\varphi(x)| /|\varphi(e)| \leq 1 /(1-\epsilon / 4) \leq 8 / 7,\|y\| \leq\|x\|+|\lambda|\|e\| \leq 15 / 7$, and $\varphi(y)=\varphi(x)-(\varphi(x) / \varphi(e)) \varphi(e)=0$. So, by hypothesis, $|\psi((7 / 15) y)| \leq \epsilon$, that is, $|\psi(y)| \leq$ $(15 / 7) \epsilon$. Then, if we take $\alpha=\gamma / \beta$, we have $|\alpha|=1$ and

$$
\begin{aligned}
|\varphi(x)-\alpha \psi(x)| & =\frac{1}{|\beta|}|\beta \varphi(x)-\gamma \psi(x)|=|\beta \lambda \varphi(e)+\beta \varphi(y)-\gamma \lambda \psi(e)-\gamma \psi(y)| \\
& \leq|\lambda||\beta \varphi(e)-\gamma \psi(e)|+|\gamma||\psi(y)| \leq \frac{8}{7} \cdot \frac{5}{2} \epsilon+1 \cdot \frac{15}{7} \epsilon=5 \epsilon .
\end{aligned}
$$

But $x$ was an arbitrary element of the unit ball of $X$, so we have $\|\varphi-\alpha \psi\| \leq 5 \epsilon$.

Notice that if $X$ is a real Banach space, the above argument still holds. Also, if $\varphi$ and $\psi$ are real valued, we can choose $e \in X$ such that $\varphi(e) \geq 1-\epsilon / 4$, giving $\beta=1$, and then from the claim, either $\gamma=1$ or $\gamma=-1$. So either $\alpha=1$ or $\alpha=-1$, yielding Phelps' result, up to a constant. 


\section{References}

[1] E. Bishop and R. R. Phelps, A proof that every Banach space is subreflexive, Bulletin of the American Mathematical Society 67 (1961), 97-98.

[2] R. R. Phelps, A representation theorem for bounded convex sets, Proceedings of the American Mathematical Society 11 (1960), no. 6, 976-983.

Antonia E. Cardwell: Department of Mathematics, Millersville University of Pennsylvania, Millersville, PA 17551-0302, USA

E-mail address: antonia.cardwell@millersville.edu 


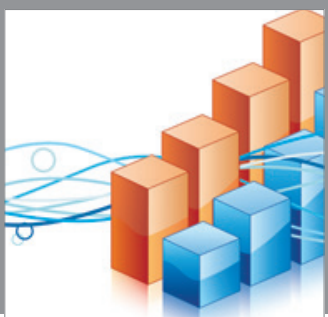

Advances in

Operations Research

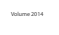

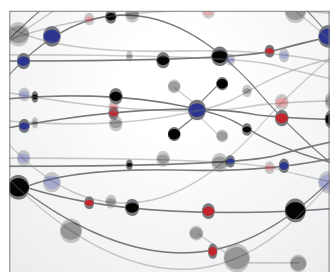

\section{The Scientific} World Journal
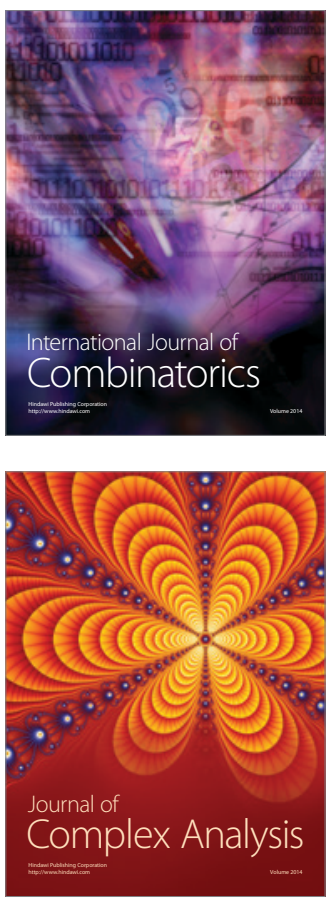

International Journal of

Mathematics and

Mathematical

Sciences
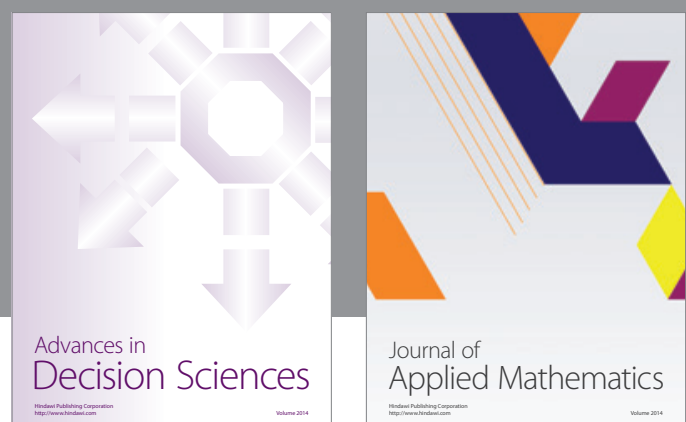

Journal of

Applied Mathematics
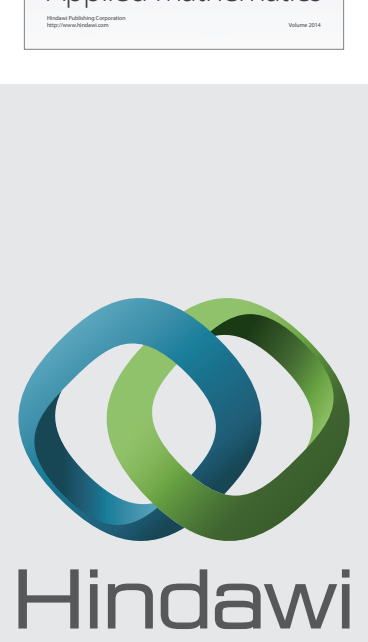

Submit your manuscripts at http://www.hindawi.com
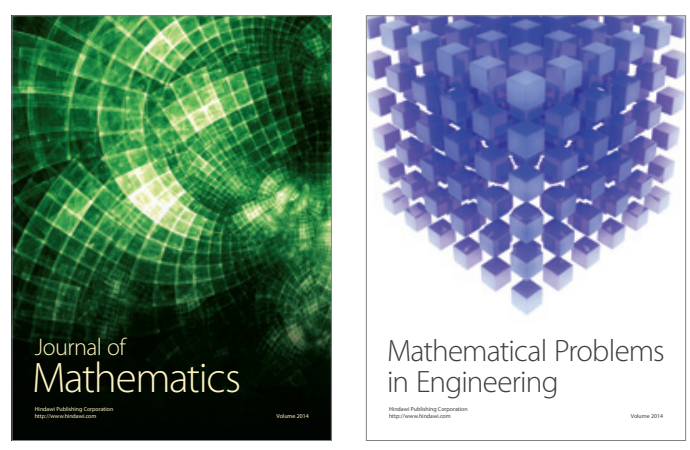

Mathematical Problems in Engineering
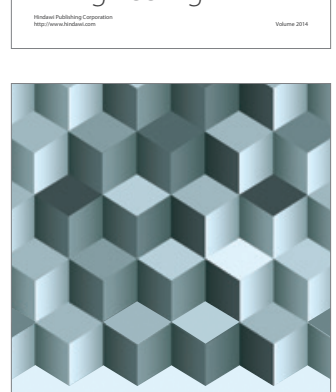

Journal of

Function Spaces
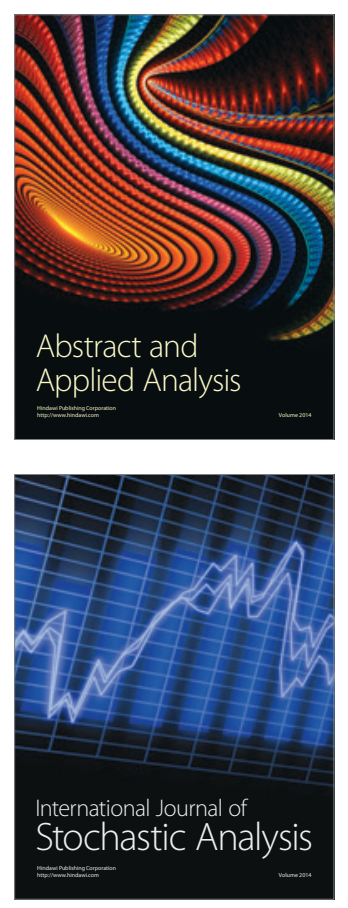

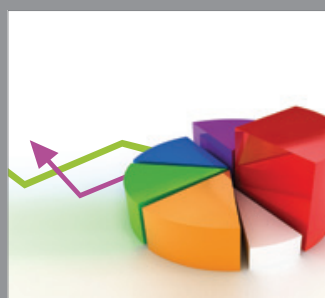

ournal of

Probability and Statistics

Promensencen
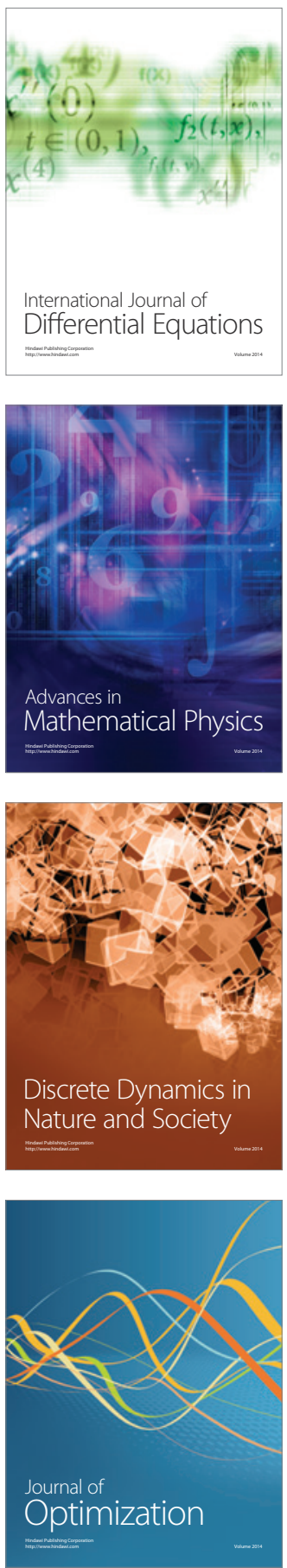